\title{
LAS CIENCIAS SU ENSEÑANZA Y SU RELACION CON LA CULTURA
}

Mary Orrego $C$.

Protesora Faculatad de Fisioterapia

Oscar Eugenio Tamayo $A$.

Profesor Facultad de Odontologia Universidad Autonoma de Manizales

Anfora

uede parecer inusual. insólito o inusitado al desprevenido lector la relación existente entre las ciencias y la cultura; sin embargo hay elementos que en forma permanente logran importantes puntos de encuentro entre estas dos esferas del desarrollo humano. El presente artículo lo iniciaremos con una breve descripción sobre lo que han sido las ciencias y su enseñanza, se resalta de paso, el vínculo existente con la cultura.

Bruner en su texto "La importancia de la educación" (1987), ubica las posibles dificultades del hombre moderno para comprender las matemáticas y las ciencias, no en la falta de capacidades, sino en que no logramos encontrar un camino adecuado y eficiente para su enseñanza. La evidencia que permite asegurar que los estudiantes de 
ciencias no aprendan realmente el conocimiento que se les "ensena" se manifiesta en forma clara en:

- Su incapacidad para utilizar los conocimientos en la explicación de fenómenos cotidianos.

- Los alumnos no aprenden los conceptos fundamentales de la ciencias sino una caricatura de ellos.

- No entienden el funcionamiento de las máquinas que usian a diario. ni son capaces de aplicar los principios de su funcionamiento.

- La enseñanza en la escuela, en lugar de ser divertida e interesante. es frustrante para el estudiante.

Dentro de las causas de mayor importancia, que explican las observaciones anteriores. encontramos el modelo pedagógico utilizado para la enseñanza de las ciencias. Ha sido un modelo en el que no se ha reconocido el dinamismo de las ciencias, no nos hemos detenido a reflexionar sobre su larga historia y mucho menos a concebir su desarrollo histórico como un elemento central para la comprensión de estas ciencias, y en forma espacial, para la orientación de su enseñanza. Ha sido un modelo pedagógico en el que se ha considerado al alumno como el şujeto que aprende. desprovisto también de historia. Esto ha conducido el ejercicio de una enseñanza genética en que trata de enseñarse «todo" en poco tiempo y se desconoce asi la experiencia del estudiante. De igual manera la enseñanza tradicional de las ciencias pretende explicar su estructura lógica actual, sin hacer evidente el engranaje conceptual que la hace posible; esto dificulta su aprendizaje.

El modelo pedagógico al que hacemos referencia, ha dado especial énfasis a la observación como act1vidad dinamizadora del aprendizaje. Dentro de esta concepción se pretende crear sucesivamente en el estudiante in proceso de impresión sensible y de abstracción. en el que el espíritu del niño (en el principio de su existencia), es una especie de tabla sobre la que se graban pro-

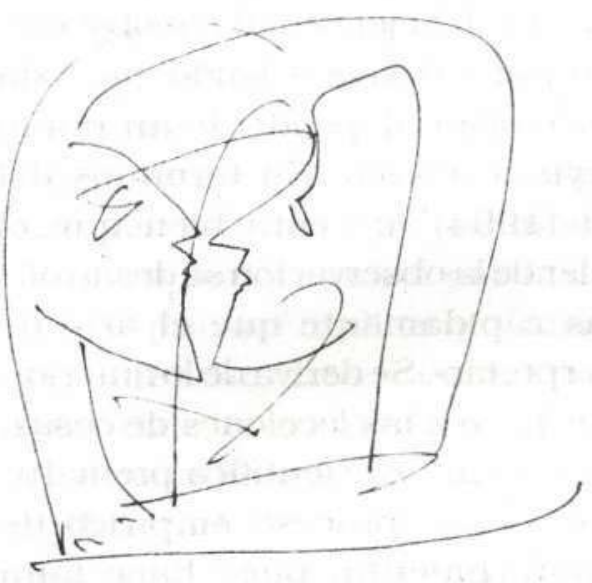


gresivamente las impresiones suministradas por los sentidos. Lo único que varía de un sujeto es el grado de sensiblilidad, es decir, la capacidad para extraer los elementos comunes a las diferentes imágenes, denominada capacidad de abstracción (Aebli. 1958).

Los métodos basados en la observación tienen un inconveniente de que sólo captan la apariencia externa de los fenómenos. Para la formación de una noción se requiere además de la observación, la actividad mental orientada hacia la construcción de un sistema de operaciones y relaciones a partir de las cuales se define la noción.

Orientar la enseñanza de las ciencias por procesos de observación es privilegiar ésta sobre la comprensión; es desvincular el aprendizaje de una parte respecto al todo que la globaliza; es pretender objetividad a través del ejercicio de la observación pura, desconociendo que toda observación depende de un punto de vista teórico. En términos del Not (1994) «no está bien que el poder de la observación se desarrolle más rápidamente que el arte de interpretar». Se deriva de lo anterior, el rechazo a las lecciones de cosas, a la enseñanza científica prematura y a todo proceso empírico de descubrimiento, pues hace falta

\section{Orientor la enseñanza}

de las ciencias por pro-

cesos de observación es

privilegiar ésta sobre la

comprenston ...

marcos de referencia sólidos para interpretarlos; queda claro que «no hay progreso para ningún escolar del mundo ni en lo que oye ni en lo que ve sino en lo que hace. Todo aprendizaje se adquiere a base de actividad» (Not. 1994) teniendo presente que la actividad a la que aquí se hace referencia, es de tipo mental.

Dentro de esta perspectiva tradicional de enseñanza de las ciencias, se diferencia con facilidad los poseedores del saber y los de la ignorancia. Entre unos y los otros -maestros y estudiantes- existe transmisión unidireccional del saber, dejando a un lado la posibilidad de ver la educación como un intercambio cultural.

El fortalecimiento de pedagogías activas en las que se oriente el proceso de formación reconoce que, aspectos tan importantes como: los 


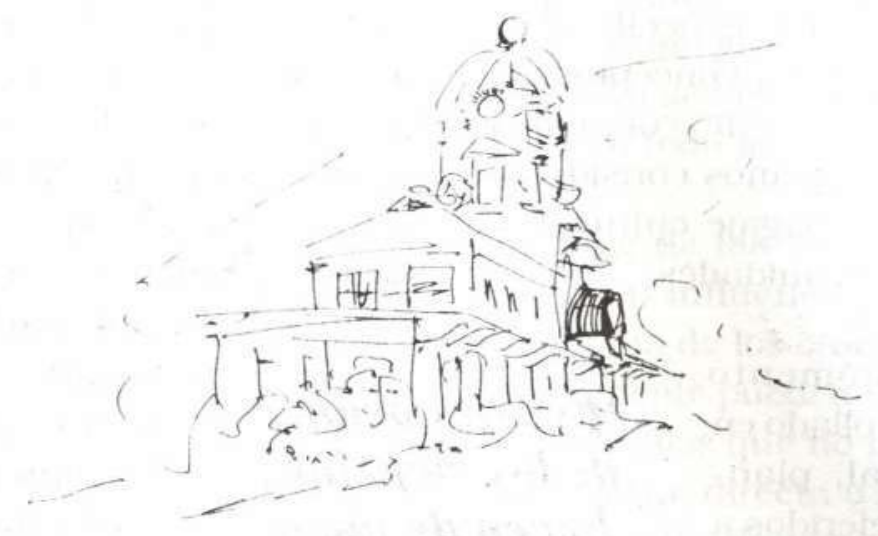

aprendizajes previos de los alumnos, el desarrollo del espíritu científico, el arte, la creatividad, y las habilidades para la resolución de problemas, son de íntima importancia en la formación de nuevas generaciones de personas en las que se privilegie la crítica frente a la memorización irreflexiva. Para el logro de este gran objetivo de la educación se requiere un maestro diferente, un maestro que logre conjurar en su acto educativo los aspectos cognitivos básicos en los vivenciales, en pos de cumplir con el objetivo que se propone. Debe ser un maestro que no esté orientado totalmente por lo científico-tecnológico, haciendo a un lado lo social y lo personal. Debe ser un maestro que desde su especialidad tenga habilidad de entablar negociaciones culturales con sus alumnos, que reconozca las dificultades de cada uno de ellos. Este maestro deberá saber elegir las tareas de aprendi- zaje para estudiantes según se estado de desarrollo, y concebir así la educación como un proceso de crecimiento y maduración permanente en el cual lo aprendido sea más significativo para el alumno.

Si bien podemos establecer diferencias entre la complejidad de las teorías, leyes, conceptos y principios, enseñados en los distintos niveles de la educación, no ha sido tan claro el interés que ha motivado su enseñanza, pues se ha orientado por la transmisión de conocimiento enciclopédico, de ésta manera se hace a un lado el que sería el objetivo central de la enseñanza de cualquier ciencia: el desarrollo de una estructura conceptual a partir de la cual se puedan explicar y comprender fenómenos cotidianos. Derivando de lo anterior y con base a los actuales condiciones de desarrollo, tanto de la ciencia como de sus formas de divulgación, se exige 
un minimo de comprensión de términos y conceptos específicos de ésta. Son términos y conceptos que ya se constituyen como cotidianos. y como tales debemos considerar; hace parte del bagaje cultural de personas y comunidades.

Hasta el momento hemos desarrollado en forma general, planteamientos referidos a la enseñanza en forma general, planteamientos referidos a la enseñanza de las. ciencias, centrados en el tipo de actividad mental que es. característico de cada uno de estos modelos. El primero orientado por procesos observacionales en los cuales la actividad del estudiante está siempre definida por el profesor; el segundo por un tipo de actividad mental en la que se privilegia la interacción que se genera entre el profesor y el estudiante, y de igual manera la interacción generada entre ellos dos y el conocimiento. A continuación discutiremos algunos elementos relacionados con la cultura y sus posibles vínculos con la ciencia.

No es nuestro propósito realizar una conceptualización amplia acerca del término "cultura» ni de las muy diferentes acepciones de que ha sido objeto. Tampoco se tiene por objetivo entrar en polémicas de orden antropológico o sociolinguístico, las cuales son de gran interés, pero rebasan con creces la intención central del presente documento. Sugerimos tomar el término cultura en sentido amplio, más cercano a los diferentes ambientes en los que nos desenvolvemos cotidianamente. Dentro de esta perspectiva, como ciudadanos, estamos siendo bombardeados permanentemente por los diferentes avances científicos, tecnológicos y culturales de la humanidad. El gran avance de ciencias como la química, la física, la astronomía, la biología, la informática, las comunicaciones, hacen de nuestra convivencia cotidiana un espacio en el que de una $u$ otra forma estamos en contacto con los productos de la ciencia y la tecnología; tal es el caso del uso del computador, de las telecomunicaciones, de la ingeniería genética, etc.

En las condiciones actuales de nues- 
tra sociedad surge la urgente necesidad de realizar un acercamiento cualitativo de los diferentes grupos a aquellos avances que de alguna forma influyen más sobre su cotidianidad, pero no como un solo elemento informativo, sino como una futura condición de sobrevivencia. Hoy en día es tal el avance científico-tecnológico que se hace necesario compartir entre los diferentes grupos un acervo de conocimientos básicos que permitan acercar el hallazgo científico «al común de la gente». Pueden citarse algunos casos ejemplificantes de la necesidad de descentralizar el conocimiento científico como son:

- La incursión del concepto «vacuna» dentro de los diferentes grupos sociales y su uso generalizado encaminado al mejoramiento de la calidad de vida de los ciudadanos.

- El surgimiento de la electrónica y, con ella, el de las calculadoras, computadoras, sistemas de almacenamiento de información, etc., si bien su desconocimiento puede no amenazar la supervivencia de las personas, sí es hoy imprescindible al interior de los diferentes grupos sociales; es cuestión de tiempo el que se generalice a toda la comunidad.

- El auge de las telecomunicaciones y su influencia sobre la vida cotidiana de los ciudadanos. Hoy dificilmente pueden ubicarse grupos sociales que no hayan tenido influencia, directa o indirecta, de algún medio de comunicación. En este sentido es planteado por Toffler como una revolución del presente
Hoy diffeitmente pueden ubicarse

grupos soctales

que no havon tent do infuencia. drecta o indirecta. de atgun medio de tomuntcacton siglo, sólo comparable con las grandes revoluciones de la humanidad.

- Grandes hallazgos de la ingeniería genética, la biología molecular y la biotecnología, han acercado gran cantidad de conceptos al ciudadano común, algunos de ellos son: fecundación artificial, control de la natalidad, herencia.

- El surgimiento de sistemas de códigos al interior de los diferentes campos de las ciencias, los cuales tienen como finalidad informar y / o prevenir al ciudadano sobre aspectos relacionados con dicha ciencia. Algunos ejemplos son los 
códigos empleados en las diferentes ramas de la ingeniería, los usados para las sustancias químicas, los usados en los sistemas de salud.

- El gran auge que en la actualidad tienen los programas encaminados a preservar la biodiversidad y proteger el medio.

Con lo anterior queda claro que la ciencia forma parte importante de la cultura contemporánea y, como tal. no es simplemente una ordenación de conocimientos acerca de la naturaleza, es una actitud mental que se adquiere $y$ debe potencializarse (Padilla, 1989). Debemos reconocer que vivimos en un mundo en el cual el conocimiento y sus múltiples aplicaciones se han convertido en elementos centrales del desarrollo de la sociedad (Misión de Ciencia Educación y Desarrollo, 1994). Hoy se plantea la urgente necesidad de integrar la ciencia y la tecnología a la sociedad y a la cultura colombiana con el fin de que ellas dejen de ser factores extraños a estas últimas. Este proceso llamado endogenización de la ciencia, debe propiciarse a todos los niveles del aprendizaje (el básico, el medio y el universitario), y enfatizar en la necesidad de aportar al desarrollo de la inteligencia como el factor más importante para el cambio y el desarrollo del país.

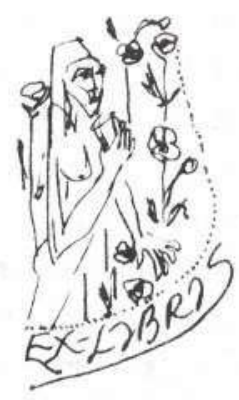

De igual manera se hace imprescindible la apropiación de la ciencia en un sentido social amplio, para lo cual se requiere crear las condiciones para que la ciencia y los procesos de progreso que ella puede generar, formen parte inseparable de la cultura nacional. Implica lo anterior, pensar en la necesidad de acercar la ciencia y sus productos a sectores cada vez más amplios de nuestra sociedad. Para tal efecto la misión recomienda el establecimiento de un «Programa Nacional de Apropiación Social de la Ciencia y la Tecnología». En este mismo sentido recomienda, entre otras acciones, conformar los centros de popularización de la ciencia, el establecimiento de museos interactivos en ciencia y tecnología, fortalecer las actividades científicas juveniles (expociencia juvenil), establecer programas de educación informal en ciencia y tecnología, así como de perfeccionamiento docen- 
te y el fortalecimiento de la investigación en pedagogia. En este sentido en la actualidad, la importancia del reconocimiento y caracterización de los preconceptos (1) de los individuos en ciencia y tecnología, radica en que hay que fundamentar el diseño de los programas curriculares, las estrategias de enseñanza-aprendizaje y la formación de los docentes de ciencias, debido a que las preconcepciones influyen en el aprendizaje, en la formación cultural, en la forma de ver y adaptarse a la tecnología moderna (Solis Villa, 1984).

Dentro de esta perspectiva de apropiación social de la ciencia a niveles formal, no formal e informal, cobra fuerza la reflexión acerca de la enseñanza de las ciencias, aspecto desarrollado anteriormente, con sus

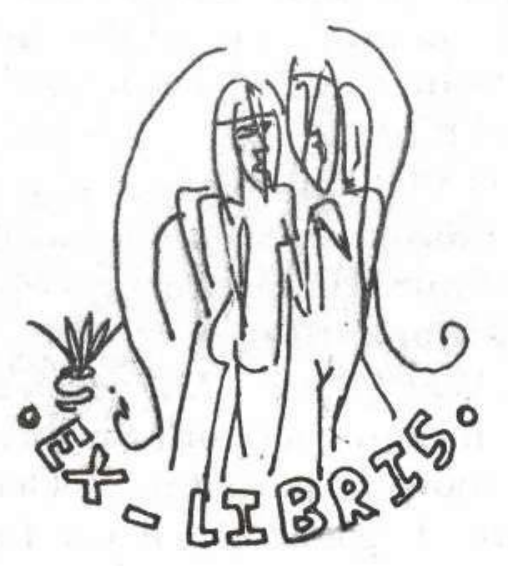

diferentes especificidades y a partir de la cual es posible acercarnos al anhelado espíritu científico, el cual favorecerá la apropiación crítica del conocimiento científico así como la generación de nuevas condiciones y mecanismos propiciadores de nuevas actitudes ante la clencia y el conocimiento. Desde esta perspectiva, Delval (1983) ubica la tmportancla de la enseñanza de las clencias y resalta a su vez elementos científicos como culturales en la medida en que:

- para podernos desenvolver en el mundo necesitamos valernos, en alguna medida de los avances científicos y tecnológicas.

- la práctica de la actividad científica es un excelente entrenamiento en el pensamiento lógico y por lo tanto contribuye al desarrollo intelectual de los alumnos.

la ciencia es además una forma de racionalidad, una forma de entender el mundoy desde ese punto de vista, tiene un valor que desborda la adquisición de unos conocimientos concretos.

(1) Los preconceptos podemos definirlos como conceptos que tienen las personas sobre algún tema y que diflere del concepto aceptado científicamente. Los preconceptos 
acerca del mundo. son elaboraciones de la realidad a partir: 1 . del uso combinado de induccion-deducción-intuición e imaginación de los individuos. 2-del uso de términos cientificos incorporados al lenguaje común. 3- de la influencia del entorno.

Los preconceptos se caracterizan por: 1 - no son congruentes con los conceptos, leyes y teorías científicas. 2 - constituyen un esquema conceptual coherente en la persona. 3 - son resistentes al cambio incluso después de mucho tiempo de educación formal. 4 - interfieren en el aprendizaje de las ciencias. siendo responsables en parte de la dificultad para aprender ciertos conceptos científicos.

\section{BIBLIOGRAFIA}

AELI. Hans. Una didáctica fundada en la psicología de J. Piaget. Buenos Aires: Kapelusz, 1987. 190 p.

BRUNER. Jerome. La importancia de la educación. México. Paidos. 1987. $199 \mathrm{p}$.

INSTITUTO COLOMBIANO PARA EL FOMENTO DE LA EDUCACION SUPERIOR. Convocatoria a la creatividad. Bogotá, 1992: ICFES.

DELVAL, Juan. La formación del espíritu científico en el niño. Uni- versidad Autónoma de Madrid. 1983. $50 \mathrm{p}$.

HELLER, Agnes. La estructura de la vida cotidiana. En: aportes Nos. 24-25. 1986. P 113-136.

COLOMBIA MISION DE CIENCIA, EDUCACION Y DESARROLLO. Colombia al filo de la oportunidad. Bogotá: Magisterio, 1995. 179 p.

LAST. Arthur. Chemistry and popular culture. En : Journal of Chemical. Education Vol. 69. No. 3. March 1992. P. 206-208.

NAKHLEH, Mary. Why some students don t learn chemistry. En: Journal of Chemical Education. vol. 69. No. 3. March 1992. P 191-196. NOT, Luis. Las pedagogías del conocimiento. Santa Fe de Bogotá. Presencia, 1994, 495 p.

PADILLA, Jaime. Estado actual y perspectivas de la investigación en ciencias blasicas. E: Ciencia, tecnología y desarrollo.Vol. 7. No. 4. cot-dic 1989. P 467-480.

STAVENHAGEN, Rodolfo. La cultura popular y la creación intelectual. En: Aportes, No. 28. 1987. P 17-22.

SOLIS VILLA, R. Ideas intuitivas y aprendizaje de las ciencias. En : Enseñanza de las ciencias.Vol. 2 No. 2, Junio 1984. P. 83-89.

VILLAVECES, C. J. L., Ciencias en un contexto de apertura. En: Ciencia y tecnología para una sociedad abierta. Bogotá: Colciencias, 1990. 